\title{
Application of Automatic Choreography Software Based on Virtual Technology in the Gymnastics Teaching
}

\author{
http://dx.doi.org/10.3991/ijet.v11i05.5692 \\ Yingbao Zhou \\ Nanjing Xiaozhuang University, Nanjing, Jiangsu, China
}

\begin{abstract}
- this research designed simulation system of gymnastics automatic choreography software. To successfully design the gymnastics movements, experiment adopted solutions based on key frame spline interpolation calculation which managed to solve the problem of location deviation; quaternion interpolation algorithm was adopted to solve the rotation problem of the subject and the body, which made the modified action by computer auxiliary choreography system completely returned to virtual figure animation. In addition, for coordination of dance and audio, this paper established movement fragments library based on sentiment types, proposed viable approach of synchronized audio and video model. Teaching experience was conducted in the gymnastics course of physical education at the University and achieved good results.
\end{abstract}

Index Terms-choreography software, gymnastic courses, multimedia teaching, virtual technology

\section{INTRODUCTION}

With the continuous development of virtual reality (VR) technology in multimedia technology, simulation teaching system in gymnastics starts to be favored by PE teaching staff and computer technology researchers [1]. Multimedia PE simulation system is able to reproduce the teachers' training intentions, thoughts and training process through computer simulation technology, designing special physical movements allow us to offer advanced and scientific training approach to teachers and students, to expand the application range in teaching and finally to forecast, analyze, evaluate, and organize sports events [2].

There are many researches on multimedia teaching system and PE teaching. And virtual reality technology is applied in various areas in sports. Many Chinese and international scholars have studied the technology in competitive sports and public fitness, and have made significant achievements in the field. Kuo et al. [3] proposed a framework, in which, the elements of the system were illustrated by highlighting the emotional impacts, which were followed by each feedback during training. Some researchers [4] studied the application of virtual technique in handball, the goal of this study is to determine which of these technologies may be preferentially utilized to analyze visual information uptake during a sport situation. These findings suggested that the analysis of visual information uptake for handball goalkeepers was better performed by using a 'virtual reality'-based methodology. Lv [5] and Zhu [6] both studied some aspects of applications of virtual technology in dancing, sports, gymnastics. They both agreed that the virtual technology conforms to the characteristics and development direction of the course, provides a more intuitive and easier learning form for students and makes the teaching process better. At present, the traditional ways of Chinese virtual human animation technology is for teachers to manually mark the movement frame-by-frame, which causes heavy workload, high cost of motion capture and insufficient accuracy [7]. Therefore, the author studied the existing automatic choreography technology in the expectation of providing $\mathrm{PE}$ teachers training approach that is completely different from traditional ones, hoping to liberate teachers from heavy manual labor and liberate students from outdoor sports venues.

Gymnastics is an elegant and graceful multiplayer game. it usually consists of several or dozen players. Designing and practicing gymnastics is very tedious and time-consuming because of the following: gymnastics venues arrangements require a lot of manpower and material resources and rehearsal needs professional technical guidance and correction for a long period of time. This study utilized automatic choreography software technology to design the movement, music and pace before the training, and conducted cell decomposition. In the teaching process, integration of teachers' intention, thoughts and conception forms a multimedia courseware that contains movements, music rhythm and explanation, so as to raise efficiency, improve the quality of arrangement and training.

\section{Automatic ChOREOGRAPHy SOFTWARE AND MODULAR DESIGN}

Automatic choreography system is divided into user layer, tools, and data tier from top to bottom. The gymnastics designer should first select the soundtrack according to its gymnastics type while designing gymnastics movements, and specify "difficulty action" at key beat in the soundtrack, the system will start "action search" module according to the designer specified "difficulty action" at back stage data tier to integrate the movements and combine series actions into coherent simulation performance, in the meantime, the system will also automatically integrate movements pieces between soundtracks to achieve smooth connections.

After completing a series of movements and music design, gymnastics designers are authorized to preview the designed movement and modify the design. Final gymnastics movements and soundtrack will be saved and generate the corresponding file for future teaching utilization and display. (See figure 1 and 2) 


\section{A. Music selection and beat settings}

Experimental choreography software database allows authors to upload their own music files to meet the needs of more types of gymnastics and rhythm, all music files in the database are in MP3 format. After uploading music files, the system will collect theme music and break up period to find the beats of the music, so that it can coincide with every movement of gymnastics.

(1) Theme collection

Considering the differences between master tracks and accompaniment tracks, this paper modified some existing parts of the BP neural network and designed classifier to locate the audio track. Design requires that: input interval values for classifiers should be: $0,1,3,4,5,6,8,10,12$, and network output should be in the interval of $[-2,2]$, when the output value is negative, the system will take the track as accompaniment, otherwise it will be considered as master track.

\section{(2) Period segmentation}

According to the existing automatic identification technology of emotions [8], this choreography software will divide the whole music into sections of different lengths. In this design, the system will extract 11 basic musical characteristics from each piece of music, and reduce preliminary dimensions according to the required quantitative description to obtain 8 formal quantitative characteristics. According to the improved gene expression programming of dynamic mutation operators [5], automatic segmentation of different types of music can be ultimately achieved through eight input and single output functions.
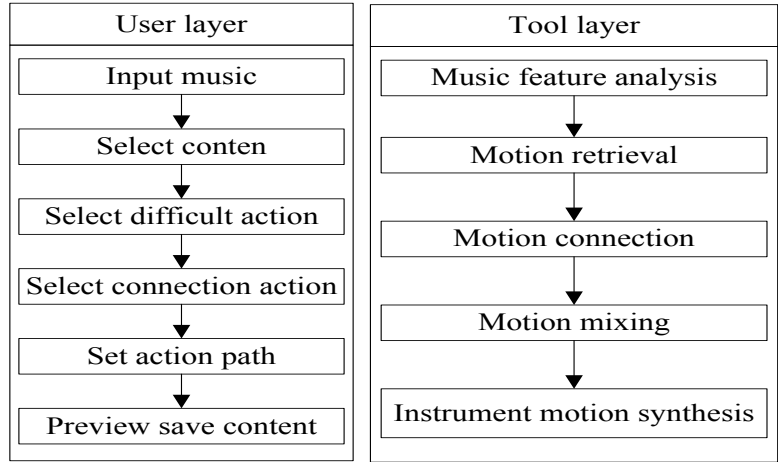

Figure 1. Choreography Design Chart of Gymnastics Automatic Choreography Software

\section{B. Motion database design}

Motion database in this paper includes two kinds of motions: difficulty actions and connecting movement. Graph node used in this paper is a key frame and diagram nodes in this automatic choreography software will select difficulty movement as the choose point for continuous movement. The default design of the software are as such: whenever the "difficulty movements" of any kind such as jumping, turning, balancing or reversal appears, the software will design a corresponding key frame. System will save after key frame selection, followed by the connection between key frames. (see figure 3)
Rope, ring, ball, rod, belt

\section{Data layer}

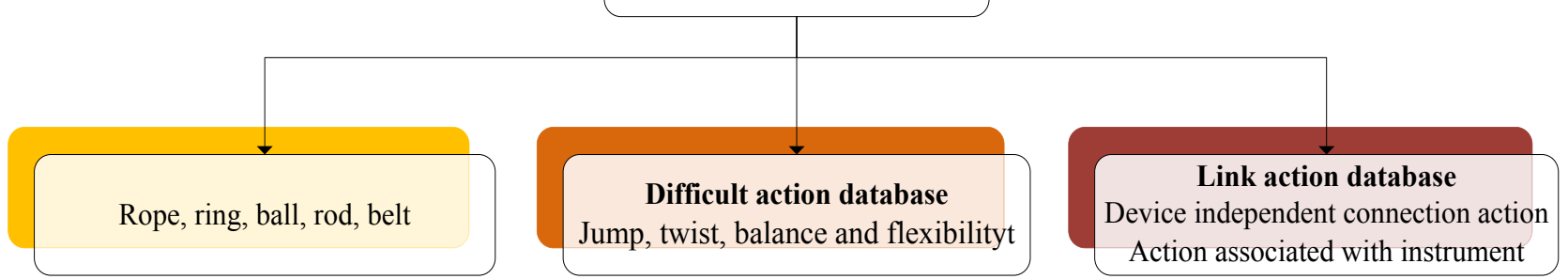

Figure 2. Database Structure Diagram of Gymnastics Automatic Choreography Software

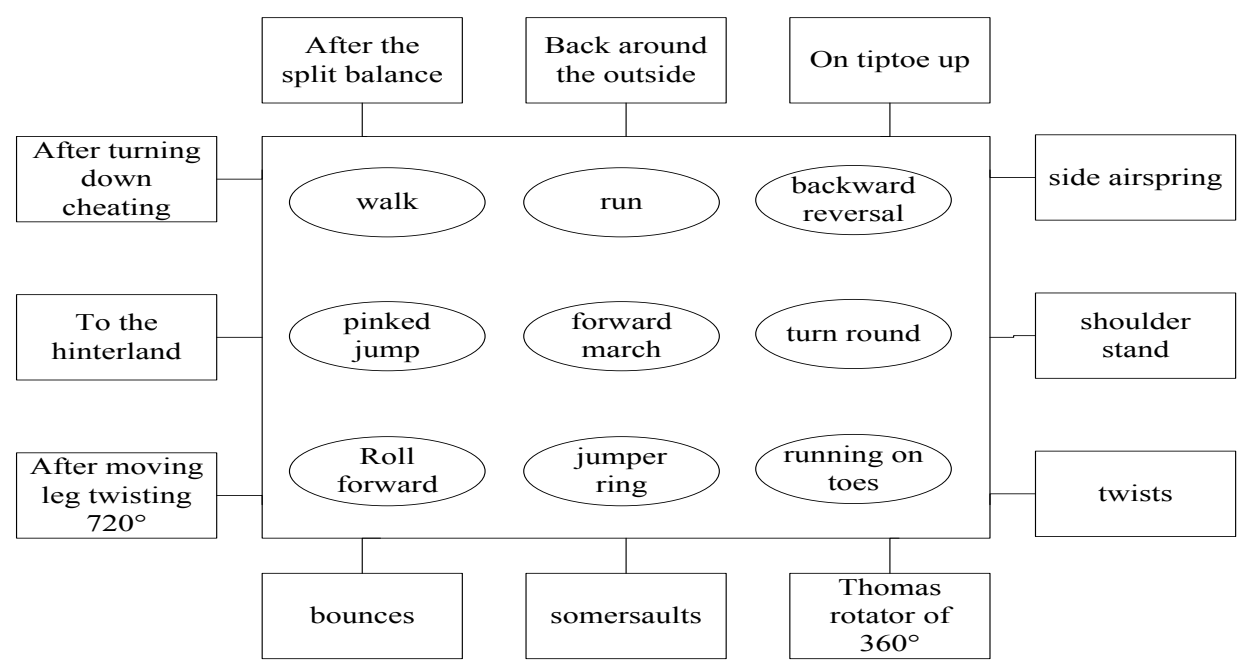

Figure 3. Movement Key Frames 
As shown in the following figure, each line represents the connection between key-frames. The connection type is divided into two kinds, including: free exercise connection and action connection involved with equipment (ropes, rings, ball, clubs, ribbon). Theory of choreography software is as follows: difficulty movements selected by gymnastics designers can be demonstrated through the system automatic connection. As shown in the following illustration, the database includes all movements required in gymnastics courses in colleges and universities, in rectangular box it's the major pattern or difficulty movement, and in oval box it's 9 most common supporting connections action or action sample. Actions within the database are through the difficult actions and secondary actions for connected and smoothed for display.

\section{Motion search module}

Motion search is for tracking initial position and motion positions of the actions and calculations, including offline and real time. Offline component is to retrieve "difficulty movement" from the real motion library and calculate its appearing time and end time, then the choreographer will, through subjective judgments, decide motion clips that can be connected to "difficulty movement" at both ends, this process is also known as "connection." Real time search only need to do depth or breadth search in accordance with point and line connections already established on the action "map". Real-time part is to select the appropriate vertices and edges from the established digraph on the basis of difficulty movement choosing by the choreographer, to add calibrated difficulty movements and connecting actions to the directed graph.

This design adopts method based on Quaternion [9] to define the distance between frames:

$$
\mathrm{D}(\mathrm{t} 1, \mathrm{t} 2)=\|\mathrm{m}(\mathrm{t} 1)-\mathrm{m}(\mathrm{t} 2)\|=
$$

In the calculation, ai, $\mathrm{i}=1,2, \ldots, \mathrm{z}$ refers to the importance of the i-rd joint of the body, is a positive number, qi(t1) and qi (t2) are two quaternions, d(qi(t1) and qi (t2)) represent the distance, distance between $\mathrm{t} 1$ and $\mathrm{t} 2$ can be calculated according to the above calculation. As can be seen by the formula, smaller distance between means easier movements. By calculating the distance between "difficulty movement" frame and "original movement" frame, we can find the position of "difficulty movement" in the "original movement".

\section{Motion synthesis}

After completing the basic search, the choreographer will begin to calibrate points and edges in the action graph and connect all corresponding points and edges between key frames, and connect all movements fragments involved in the gymnastic, complete the motion path according to the specified initial position.

Space coordinate transformation will have to be introduced to convert all run path of gymnastic action graph into smooth curve. In choreography software design, the position of the root node of the simulation movement is in a two dimensional plane $(\mathrm{x}, \mathrm{z})$, while the coordinate records three dimensional positions of simulation movements. Thus when connecting the initial and end points, the system will multiply the motion of points with transformation matrix $\mathrm{T}, \mathrm{T}$ is the identity matrix. When the movement ends at one edge and begins to enter the next edge, multiply $\mathrm{T}$ with $\mathrm{T} \theta$, the corresponding transformation matrix when the two adjacent edges align, $\mathrm{x} 0, \mathrm{z} 0$ will be updating accordingly. Among them, $\mathrm{T} \theta, \mathrm{x} 0, \mathrm{z} 0$ refer to the matrix in the $\mathrm{XZ}$ plane formed by $\theta$ degrees rotation around the $\mathrm{Y}$ axis and translation $(\mathrm{x} 0, \mathrm{z} 0)$.

\section{E. Motion Drive}

Motion drive is to produce motion presentation sequences with simulation effect by motion editing algorithm in accordance with the design of initial actions. The technology not only can show lifelike effect, it also allows designers to modify the motion module, which is conducive to human-computer interaction. Generating new motion sequences in the automatic choreography software includes the following three basic items:

(1) Detailed and thorough information on target motion. Detailed information on choreographer's expected movements, such as initial location coordinates, end position coordinates and other details;

(2) Allows movements connection. Uploaded movements allow motion capture and connection;

(3) Smooth transitions, allows modification if final animation does not meet the mechanical requirements or collision needs.

\section{F. Dance and sound coordination}

Traditional audio driving video technology can drive movements through music, it provides a broad platform for art education. In the meantime, application of virtual reality technology makes the teaching of art more simple and convenient. This paper designed a coordination scheme of sound and dance that can be applied in gymnastics teaching and presentation, it consists of three parts:

\section{(1) Music library}

Music library accepts files uploaded by designer, sorts them out and divides them into segments, and then save them. Gymnastic choreographer can select music that fits their design style within the database, or they can also upload music files.

\section{(2) Movement library}

Movement library stores plenty movement keyframes, these keyframes correspond to different modes of musical emotions and movements. With reference to detailed information of movements key frames, choreographer can select and combine music files with the appropriate movements and demonstrate continuous gymnastic movements.

\section{(3)Emotion - movement matching table}

Emotion - movement matching table in the database can be used directly. However, music files and movement keyframes uploaded by the designers will have to match the emotions with movement again. In order to ensure harmony and smoothness of music and movements, the design is using the most standard glossary Hevner [10] in the industry, which converses all the music emotions into the eight basic emotions and assigns the rhythm. And the most basic common movements in the gymnastic movements database are classified according to the glossary resume Poser library (Smith MicroSoftware, Watsonville, California), eventually establishes a link between music/movement script files.

\section{G. Automatic choreography software interface}




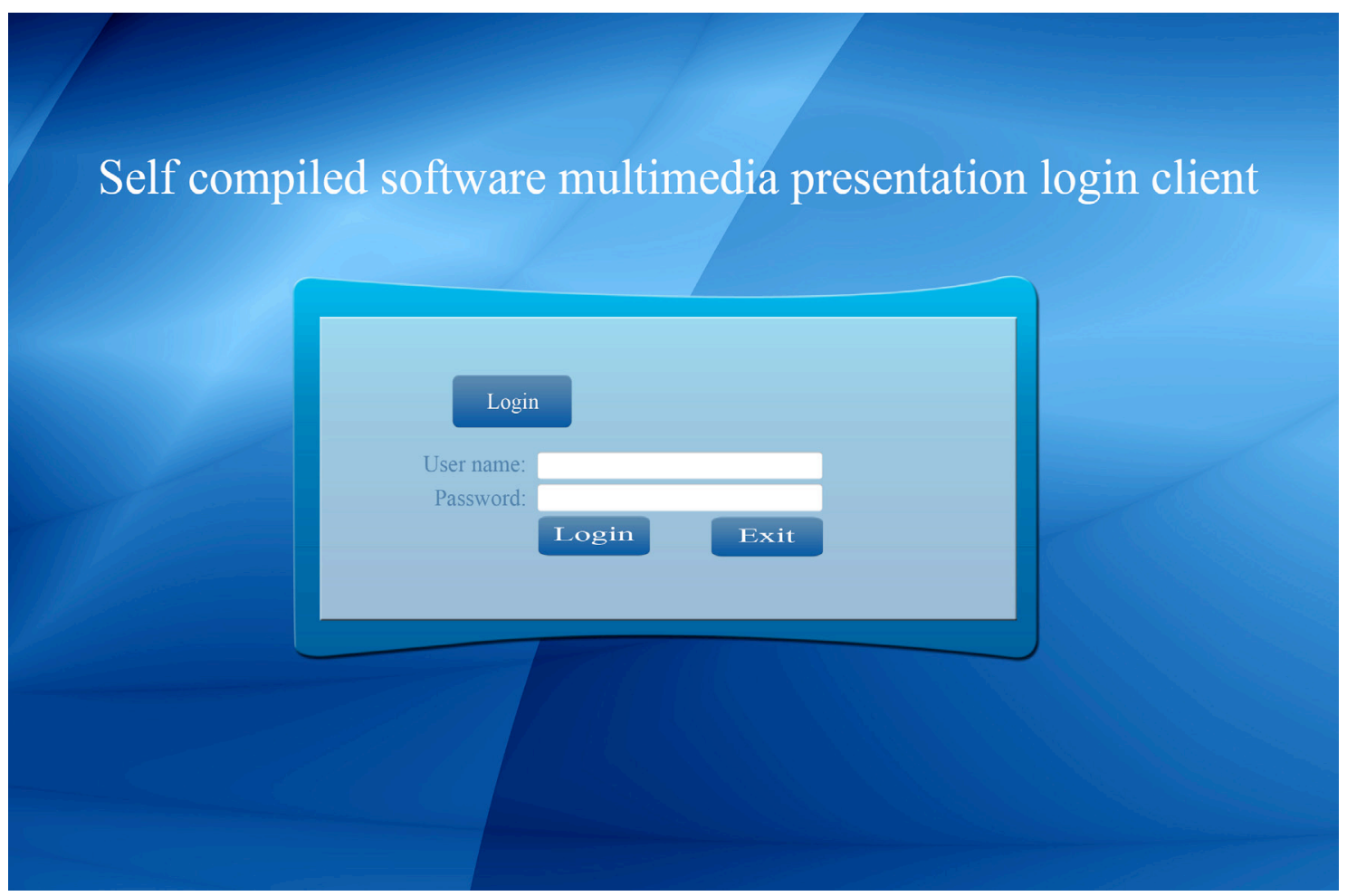

Figure 4. Multimedia Demo Login Client of Automatic Choreography Software

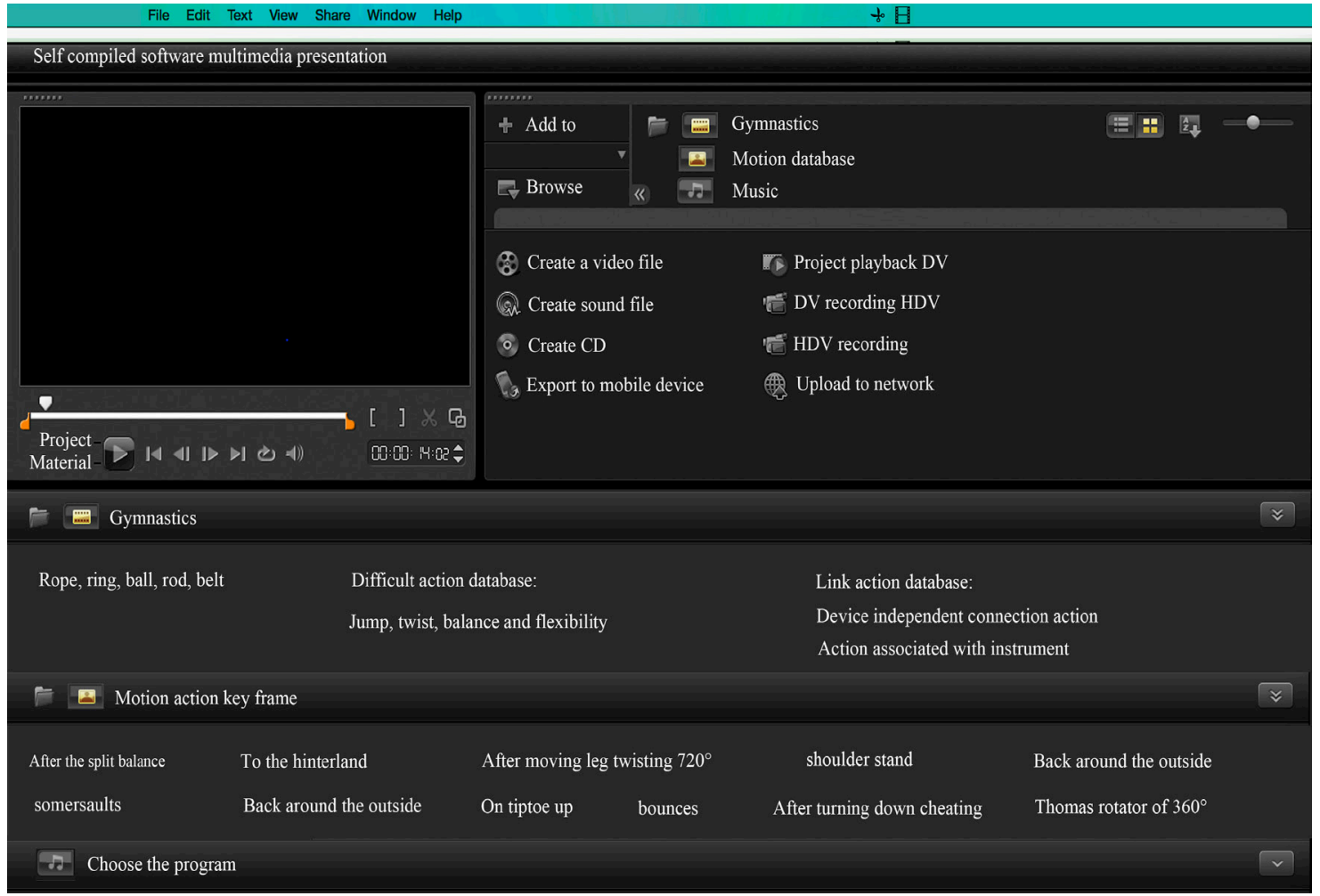

Figure 5. Automatic Choreography Software Designing Gymnastic Movements 


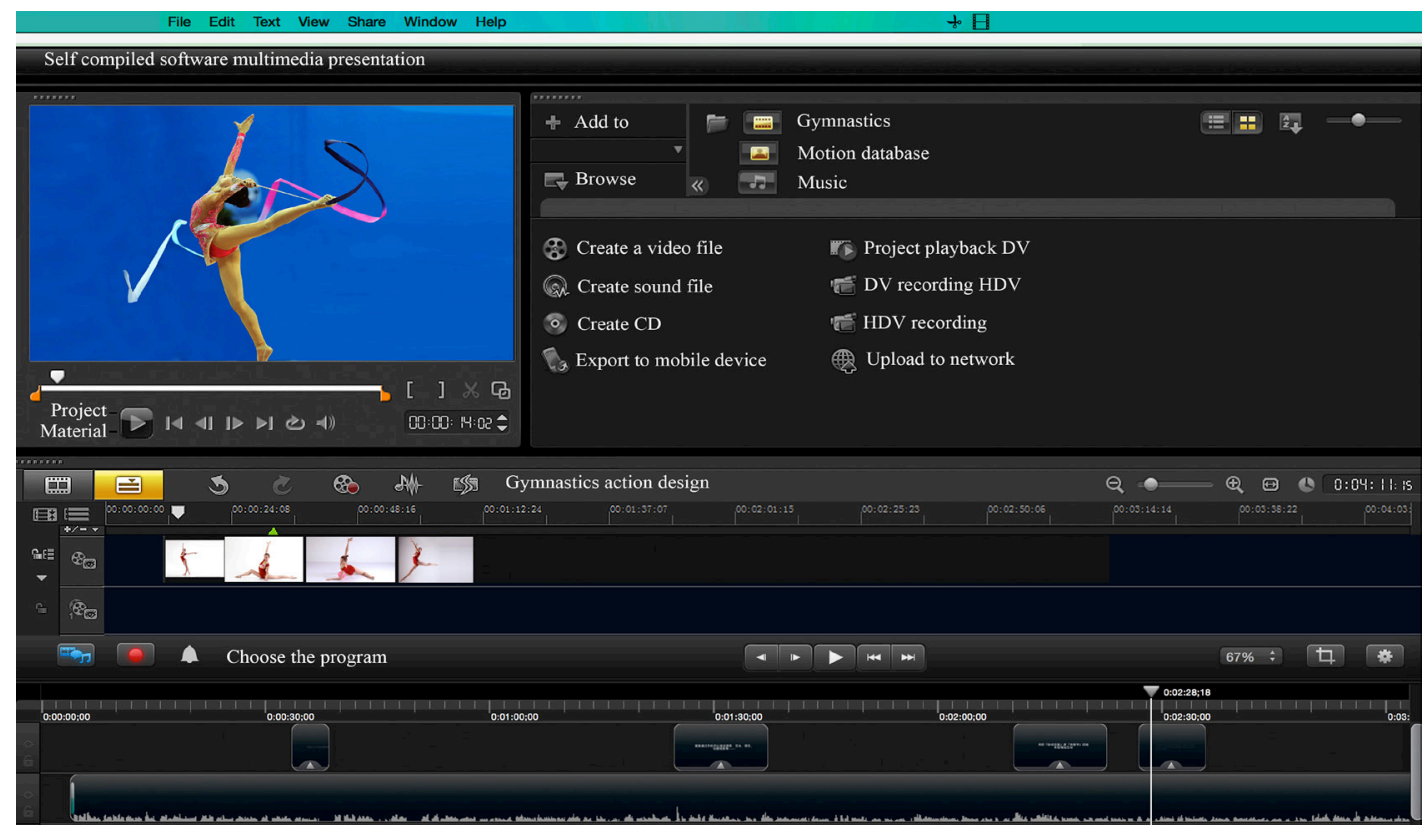

Figure 6. Gymnastic Movement Storage

\section{Multimedia Teaching EXPeriment Of The GYMNASTICS COURSE}

\section{A. Experiment subjects}

Randomly select 80 PE students (including 40 boys and 40 girls) as the study subjects. 40 students from class 1-4 were selected as Experimental group, 40 students from class 5-8 as Control group. Experimental group had multimedia gymnastics course with automatic choreography software, Control group had gymnastic course in the traditional way, course content and schedule were the same for the two teams, the course were one semester with 56 lesson hours.

\section{B. Learning outcome evaluation methods}

The experiment comprehensively evaluated the learning effects of gymnastic course by automatic choreography system from three aspects. First, students' application ability of the basic theoretical knowledge of gymnastic course, assessed mainly by exam $(20 \%)$; second, students' master and comprehension level of basic gymnastic movements, individual gymnastics movement as assessment criteria (35\%); third, the effects on students' performance results, continuous movement as standard (45\%).

\section{Experimental results and analysis}

After completing one semester course, evaluation of all students with the above assessment criteria were conducted, the experimental results are shown in table 1 . We can see that Experimental group got better scores than the Control group $(\mathrm{P}<0.1)$.

TABLE I.

COMPARISON OF AVERAGE SCORES BETWEEN COMPARISON GROUP AND TEST GROUP (SCORE, $\mathrm{X} \pm \mathrm{S}$ )

\begin{tabular}{lcc}
\hline & No of People (n) & Test Score \\
\hline Control group & 40 & $73.686 \pm 5.987$ \\
Experimental group & 40 & $76.029 \pm 5.994$ \\
$\mathrm{~T}$ & & -1.75 \\
$\mathrm{P}$ & & 0.0843 \\
\hline
\end{tabular}

The experiment comprehensively evaluated the learning effects of gymnastic course by automatic choreography system from three aspects. Results show that Experimental group had better application of theoretical knowledge than Control group $(\mathrm{P}<0.1)$. Experimental group had higher master and comprehension level of basic gymnastic movements than Control group $(\mathrm{P}<0.05)$. and performances showed no significant differences between the Experimental group and Control group ( $\mathrm{P}>0.1)$. Comprehensive evaluation of the Experimental group was significantly better than the Control group $(\mathrm{P}<0.05)$.

In order to understand the effect of multimedia teaching of gymnastics, at the end of the course, questionnaire survey were conducted among Experimental group where students completed the multi-media gymnastics lesson with automatic choreography software.40 questionnaires were issued and been collect back with $100 \%$ recovery rate. Results showed that $82.5 \%$ students thought multimedia teaching of gymnastics had good effect $(\mathrm{P}<0.01)$, and it would help to improve students ' learning interest and motivation, and had good grasp of learning content. But there are also some $10 \%$ students said they did not adapt to this teaching method, and had low learning interest and motivation, grasp of learning content had not been ideal (see Table III).

\section{CONCLUSIONS}

Through interactions of dance moves and combinations by the automatic choreography software, it combines, sorts, collects gymnastics movements forming series of gymnastic movement combinations by using matrix converting and editing of three dimensional model, and movement-music conversion table, to eventually build a gymnastics choreography teaching system. Experiment showed that the students ' every move can be shown in real time and in multi-dimensions, achieved transformation from experience-based training and analysis method to training and analysis method based on simulation of human motion modeling.

In order to objectively evaluate the teaching effect, exam score analysis and questionnaire investigation are 
PAPER

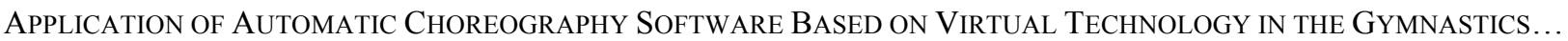

TABLE II.

COMPARISON OF COMPREHENSIVE EVALUATION ON 3 ASPECTS FROM 2 TEAMS

\begin{tabular}{|c|c|c|c|c|c|}
\hline & No of People (n) & Theoretical Knowledge & Comprehension Level & Performance Effect & Total Score \\
\hline Control group & 40 & $13.686 \pm 5.987$ & $29.413 \pm 4.666$ & $30.234 \pm 3.459$ & $73.295 \pm 8.368$ \\
\hline Experimental group & 40 & $16.029 \pm 5.994$ & $31.324 \pm 2.725$ & $30.882 \pm 3.743$ & $77.995 \pm 10.384$ \\
\hline $\mathrm{t}$ & & -1.75 & -2.24 & -0.8 & -2.23 \\
\hline $\mathrm{P}$ & & 0.0843 & 0.0289 & 0.4236 & 0.0287 \\
\hline
\end{tabular}

TABLE III.

EVALUATION ON MULTIMEDIA GYMNASTIC LESSON By STUDENTS

\begin{tabular}{|c|c|c|c|c|c|c|}
\hline \multirow[b]{2}{*}{ Questionnaire Contents } & \multicolumn{6}{|c|}{ Effect Evaluation by Students [n(\%)] } \\
\hline & $\begin{array}{c}\text { Strongly } \\
\text { Agree }\end{array}$ & Agree & Neutral & Disagree & $\begin{array}{c}\text { Strongly } \\
\text { Agree }\end{array}$ & $\begin{array}{c}\text { Chi-square } \\
\text { Test }(P)\end{array}$ \\
\hline The teaching method has good function & $14(35)$ & $19(47.5)$ & $5(12.5)$ & $2(5)$ & $0(0)$ & 0.0003 \\
\hline Adopted well to the teaching method & $13(32.5)$ & $16(40)$ & $7(17.5)$ & $4(10)$ & $0(0)$ & 0.0293 \\
\hline Good grasp of learned content & $6(15)$ & $11(27.5)$ & $16(40)$ & $7(17.5)$ & $0(0)$ & 0.1023 \\
\hline Conducive to increase interests and initiative to learning & $14(35)$ & $16(40)$ & $6(15)$ & $4(10)$ & $0(0)$ & 0.0155 \\
\hline
\end{tabular}

designed. from analysis of exam scores, we can see that the improvement effect from multimedia teaching by using automatic choreography software is mainly reflected in the "comprehension level" of unknown knowledge, of course, it also increased the total score. These results suggest: the new teaching methods tend to be more scientific, the teaching tends to be more intuitive, vivid and interesting, therefore, students tend to learn with initiative instead of passive memorization, which effectively promotes students ' understanding and application of knowledge. Second, application of automatic choreography technology in gymnastics multimedia teaching, especially its simulation on course video relieved teaching and learning from many unnecessary constraints in the process, enhanced its depth, strength and diversity of gymnastics teaching, increased teachers' teaching options, learning in the training site all day in the past has changed into combination of on-site training and multi-media classroom learning, this greatly stimulated fondness and generalization to the gymnastic teaching assist system based on automatic choreography technology from students of gymnastic and related majors, it also shows the unique charm of computer technology in sports training.

All in all, the gymnastics teaching is not only about expressing its meaning and style through body language and style, but also about empathizing with the perfect combination of dance and sound designed by virtual reality with the theatricality, and ultimately achieving improvement of comprehensive ability by taking the gymnastics courses. With the development of computer technology and interactions of science and technology with human nature, virtual reality of virtual technology will profoundly affect modern gymnastic teaching, teachers and students will be liberated from the heavy body movements, concentrate on the details of the movements, sound $\&$ dance beauty and harmony between people.

\section{REFERENCES}

[1] Pan, Z., Cheok, A.D., Yang, H., Zhu, J., \& Shi, J., "Virtual reality and mixed reality for virtual learning environments," Computers \& graphics, vol. 30, no. 1, pp. 20-28, February 2006. http://dx.doi.org/10.1016/j.cag.2005.10.004
[2] Hughes, C.E., Stapleton, C.B., Hughes, D.E., \& Smith, E.M., "Mixed reality in education, entertainment, and training," Computer Graphics and Applications, IEEE, vol. 25, no. 6, pp. 24-30, November-December 2005. http://dx.doi.org/10.1109/MCG. $\underline{2005.139}$

[3] Kuo, J.Y., Chen, C.H., Roberts, J.A, "framework for understanding the emotional impacts in virtual sport training: In this work, a framework of a virtual sport training system is proposed based on relevant studies in motor skill learning, the formats of feedback and the emotions in virtual training to address the often overlooked emotional needs of athletes in such systems," Virtual and Physical Prototyping, vol. 8, no. 4, pp. 235-239, December 2013. http://dx.doi.org/10.1080/17452759.2013.862957

[4] Vignais, N., Kulpa, R., Brault, S., Presse, D., \& Bideau, B., "Which technology to investigate visual perception in sport: Video vs. virtual reality," Human movement science, vol. 39, pp. 12-26, February 2015. http://dx.doi.org/10.1016/j.humov.2014.10.006

[5] Lv, P.F., "Automatic Choreography System Based on Emotion Tagging," Electronic technology, no. 8, pp. 18-21, October 2008.

[6] Zhu, P., "Study on Application of Network Curriculum in the Teaching of Sports Dance in Colleges," Science and Technology in Western China, vol. 10, no. 30, pp. 76-77+65, March 2011.

[7] Wang, J.J., "Differences between Elements of New Media Dance and Traditional Dance," Drama and Film Monthly, no. 5, pp. 3435 , October 2014

[8] Liu, H., "Study on Development and Application of Computeraided System on Choreography," Art Gallery, no. 4, pp. 106-108, April 2011.

[9] Ding, G.Y., Li, H.S., Huang, T.Y., Zhang, L.F., Gao, J., Zhang, D.W., "Virtual Choreography System Prototype for Large-scale Square Performance," Journal of System Simulation, vol. 20, no. S1, pp. 136-140, April 2008.

[10] Kallinen, K., Ravaja, N., "The role of personality in emotional responses to music: Verbal, electrocortical and cardiovascular measures," Journal of New Music Research, vol. 33, no. 4, pp. 399-409, September 2004. http://dx.doi.org/10.1080/0929821052 000343868

\section{AUTHOR}

Yingbao ZHOU is a Lecturer of School of Department of Physical Education, Nanjing Xiaozhuang University, Nanjing 210028, China. His research interests include Physical Education and Sports Exercise. (zhouyb111@yeah.net).

Submitted 29 March 2016. Published as resubmitted by the author 27 April 2016. 